\title{
Marchandage d'âmes entre vivants et morts
}

Bartering souls among the living and the dead

\section{Roberte Hamayon}

\section{OpenEdition}

\section{Journals}

Édition électronique

URL : http://journals.openedition.org/span/396

DOl : 10.4000/span.396

ISSN : 2268-1558

\section{Éditeur}

École pratique des hautes études. Sciences humaines

\section{Édition imprimée}

Date de publication : 1 septembre 1978

Pagination : 151-179

ISSN : 0294-7080

\section{Référence électronique}

Roberte Hamayon, " Marchandage d'âmes entre vivants et morts », Systèmes de pensée en Afrique noire [En ligne], 3| 1978, mis en ligne le 05 juin 2013, consulté le 19 avril 2019. URL : http:// journals.openedition.org/span/396 ; DOI : 10.4000/span.396 


\author{
M A R C H A D A G E D' Â E S \\ E N T R E V I V A N T S E T MOR T S \\ par Roberte Hamayon
}

I1 s'agit ici avant tout d'ajouter un spécimen à la collection de sacrifices réunie par le L. A. 221. Libre de toute armature théorique préalable, 1'exposé, consacré à la modalité bouriate du sacrifice, devra beaucoup à la spontanéitê qu'ont montrée les matêriaux eux-mêmes à répondre à l'appel.

Appel au demeurant fort modeste, reposant sur la représentation du sacrifice comme mise à mort rituelle. Malgré l'imprécision de ce signe de'reconnaissance pour la sélection des données, I'acte sacrificiel ne se laisse confondre ni avec la guerre ni avec le meurtre ni avec la quête alimentaire, ni encore avec le châtiment légal. Caractérisé par la destruction matérielle d'êtres vivants -- destruction sans caractère utilitaire apparent --, le sacrifice semble se récuser en tant que mise à mort, et se présenter comme une offrande, un support de message, une incitation à l'échange, un facteur de transformation ou de transfert. Mais à quoi bon détruire pour offrir ? Ne peut-on transférer sans avoir au préalable altéré ? Et comment se justifie un acte consistant à offrir une chose détruite ? Sans doute la notion de destruction doitelle être limitée au sort concrètement subi par la victime, et ne pas être autorisée à déborder sur le ou les aspects non concrets de ce sort. Par ailleurs, fuir l'assimilation à une destruction entrâ̂ne-t-il la promesse d'un destin posthume particulier pour les sacrifiés ? Certaines prétentions fonctionnelles du sacrifice supposent l'existence de partenaires, d'autres représentent des manipulations originales des êtres, toutes se référant implicitement à des instances symboliques (I). La cohorte des questions similaires qui ont surgi au fil de 
1 'examen des données, sans épuiser, loin s'en faut, le mystère du sacrifice, a dicté les étapes de la démarche analytique suivie ici : seront d'abord décrits brièvement les divers types de rites comportant des sacrifices, puis examinées les situations de sacrifiant (bénéficiaire), de sacrifié et de dédicataire, chacune de ces situations mettant en lumière, à sa façon, le rôle du chaman. On tentera ensuite de dégager la spécificité du sacrifice en tant que geste (d'offrande, de transfert, de rêtribution) fait à l'égard de partenaires symboliques, en tant que mise à mort, et en tant que traitement rituel d'êtres vivants.

Cousins septentrionaux des Mongols, les Bouriates en partagent, à quelques nuances près, la langue et la culture. Ils vivent de part et d'autre du lac Baïkal, en Sibérie centrale.

C'est à l'ouest, où la géographie fait alterner la montagne, la steppe et la taiga, que l'implantation bouriate est la plus ancienne. Nomades de toujours, les Bouriates $s^{\prime}$ adonnaient autrefois à la chasse et à l'élevage. A celui-ci s'est peu à peu associée l'agriculture, depuis la colonisation russe, qui a débuté au XVIIIe siècle. Dans son sillage, s'est imposée la sédentarisation qui, jointe aux nouvelles mesures administratives, bouleversa le régime clanique. Le chamanisme se trouva, quant à lui, relativement peu atteint par 1 'influence orthodoxe. En dehors du département national d'Ust'-Orda, les Bouriates occidentaux se disso1vent aujourd'hui dans une population sibérienne mélangée.

Quant aux steppes de l'est du Baîkal, peuplées en majorité de groupes issus de Mongolie, elles furent le cadre d'un pastoralisme nomade de grande amplitude, très proche de celui que connaissent les Mongols. Comme eux, ils adoptèrent, mais plus tard (XIXe siècle) et moins profondément semble-t-il, le lamaỉsme hérité du Tibet. Au nombre de 315000 , ils sont constitués aujourd'hui en une République autonome d'URSS.

$C^{\prime}$ est des Bouriates occidentaux de la fin du XIXe siècle et du début du XXe qu'il sera question ici, en raison de la nature des faits et de celle des sources. Fatale à la structure de la société à $1^{\prime}$ Ouest, l'acculturation fut fatale à la vie rituelle traditionnelle à l'Est. La littérature russe antérieure à la Révolution d'Octobre est très copieuse; une partie en est due à des Bouriates bilingues issus de l'intelligentsia qui se forme à la fin du XIXe siècle. Depuis lors, la littérature orale n'a cessé

1. II est coutumier de faire, à propos du sacrifice, usage de l'opposition sacré/profane, de plus en plus abandonnée toutefois en raison de ses embarrassantes implications, ou de l'opposition visible/ invisible. Cette dernière ne peut être retenue à propos des représentations bouriates : il arrive en effet à certains humains de voir ces autres êtres, qu'on désignera ici, faute de mieux, comme symboliques. Pour des raisons analogues, le terme de victime, qui insiste sur le préjudice subi, sera abandonné au profit de celui, descriptif, de sacrifié, lorsqu'il s'agira de définir son statut. 
d'être collectée, parfois assortie de commentaires et de descriptions de rituels. Quant à I'enquête ethnologique actuelle (2), impuissante à observer des survivances clandestines, elle permet d'une part d'atteindre des aspects considérés comme anodins de la tradition (dont des traits plus fondamentaux viennent se greffer, épars, sur ces traits prétendus anodins), d'autre part, de comprendre et d'évaluer tant la littérature descriptive que la littérature religieuse bouriate elle-même, très riche.

$$
\text { I. RITES A SACRIFICE }
$$

Il n'y a de terme spécifique ni pour le sacrifice ni pour les rites comportant un sacrifice. L'épisode de la mise à mort rituelle est désigné, comme une mise à mort ordinaire, par le terme alalga, formé sur la racine de "tuer" (alaxa). On rencontre trois types de rites à sacrifices :

a. tajlgan.

C'est un nom d'action formé sur une racine qui, en emploi ritue1, a le sens de "faire un rite de sacrifice en faveur de quelqu'un", et, en emploi profane, celui de "choyer, dorloter, courtiser". Centré sur le dédicataire auquel on donne ainsi une marque d'attention, ce terme se distingue du "sacrifier" français, centré, 1ui -- du moins dans ses emplois dérivês -- sur 1'objet qui fait les frais de cette marque d'attention, sur 1 'objet immolé.

Intimement lié à l'institution clanique, ce rituel se déroule sur le flanc d'une montagne censée avoir été choisie par les ancêtres pour y résider, et située en général sur le territoire d'estivage du clan. "Maîtres" des lieux, les ancêtres dispensent le droit de les occuper. Leur résidence a pour expression concrète une ou plusieurs pierres, de

2. Trois missions ont été effectuées en Bouriatie, au titre des échanges entre le CNRS et 1 'Académie des Sciences d'URSS. Deux d'entre elles se sont déroulées chez les groupes se rattachant à la tradition de 1'ouest du lac : en pays Barguzin, peuplé d'exirit émigrés, et en pays Tunka, au sud-ouest. 
forme et de taille variables. Ces pierres, en tant que propriété du clan et marque de 1 'espace sacrificiel, font l'objet de litiges entre clans au cas oũ change l'attribution du territoire : si l'ancien occupant revient sacrifier auprès d'elles, il porte atteinte au droit d'occuper le sol du nouvel occupant et par là à sa prospérité ; s'il emporte ses pierres, il prive son successeur des "maîtres" de son territoire (cf. Baldaev 1970, pp. 63-64).

La participation des hommes du clan est aussi impérieusement prescrite que celle de leurs épouses est interdite. S'abstenir revient à s'exclure du clan, du moins à en perdre la solidarité (3).

C'est au début et à $1 a$ fin de l'estivage qu'ont lieu les principaux tajlgan. Jalons de la vie nomade, ils resserrent les liens entre les membres vivants et morts du clan. Entre les deux pôles de la saison, viennent s'insérer d'autres tajzgan, de nombre, d'importance, de destination et d'objet variables, toujours spécialisés. Leurs destinataires, localisés sans être pour autant détenteurs de territoires, issus d'âmes de morts sans être des ancêtres, sont les "mâ̂tres" de diverses choses de la vie : activités économiques, maladies, etc. On les "courtise" au grê des calamités naturelles ou sociales. Qu'une année connaisse une sécheresse abusive, une épidémie de strangulation décimant la jeunesse (4), une épidêmie de peste đécimant le bétail, et par précaution les années suivantes, l'esprit identifié comme responsable recevra un sacrifice. Ainsi a-t-on vu, à la fin du XIXe siècle, comme une génération spontanée de tajlgan, suscités par les multiples troubles consécutifs à l'acculturation : resserrement de l'espace, dissolution des liens sociaux, banditisme, etc., crêant une atmosphère de catastrophe latente,

3. Par ailleurs, il existe des fêtes féminines qui ont des points communs avec les tajlgan (Vjatkina, p. 55) et les femmes participent aux tajlgan adressés aux maîtres des eaux.

4. Comme celles qui, dans la seconde moitié du XIXe siècle, frappèrent la commune d'ülej du gouvernement d'Irkoutsk, selon le modèle fourni par la jeune Düxe qui, malheureuse en ménage et contrecarrée dans sa vocation chamanique, s'étrangla. Belle et gaie, elle incite depuis lors les jeunes à 1 'imiter pour partager ses fantaisistes plaisirs. Cette joyeuse bande d'esprits de suicidés, au nombre de 300 vers 1890 , est censée avoir été récemment réduite de moitié par suite de participation à la 2ème guerre mondiale. 
que la répétition de précautions rituelles allège et conjure. Le clan jangut accomplissait une dizaine de tajlgan (Manžigeev, 195), le premier clan Abaganat de huit à dix, le deuxième clan Abaganat onze... (Girčenko, 74). De quoi occuper le temps de l'été et mener à la ruine.

Le sacrifice clanique d'ouverture ou de clôture de l'estivage sert de modèle plus ou moins fixe à tous les tajlgan. En voici une description schématique :

Une collecte préalable auprès des membres du clan, si éloignés soient-ils, permet de réunir les ingrédients nécessaires : animaux sacrificiels (5), alcool-de-lait (6) et autres denrées, peaux de petits carnassiers, récipients. Le rite s'ouvre au matin dans la yourte d'un vieux, par une purification des accessoires rituels et des assistants (7). On procède à une première aspersion d'alcool en faveur des ancêtres, avant que ne s'ébranle vers la montagne clanique le cortège des participants à cheval (8). Tous s'installent à flanc de montagne, dans 1'ordre rigoureux qu'impose la séniorité généalogique. Devant chaque patrilignage (9) sont disposées ses auges, et est plantée sa branche de bouleau signalètique, tandis que derrière trônent d'énormes chaudrons. A 1 'écart, vers 1 'ouest, des groupes de minces bouleaux plantés au nom de toute la communauté ; on y accroche les peaux de carnassiers (zibeline, putois,...) et on les relie entre eux par des cordes. Chaque patrilignage délègue un de ses membres pour conduire une nouvelle aspersion, accompagnée d'une prière à l'unisson et suivie de divination sur le lancer des bols vides. L'"aspergeur" (xajalgaša) doit, en principe, surtout s'il est chaman, se contenter de flairer le liquide avant de le répandre, de le goûter après, pour transmettre enfin le reste aux assistants, par rang $d^{\prime a ̂ g e . ~}$

5. Les juments sacrificielles, bajtahan, sont achetées en commun pour des prix très élevés à des familles qui en ont le monopole (Manžigeev 56, 140). Brebis et chèvres sont fournies séparément par les patrilignages. Pour le grand tajlgon du deuxième clan Abaganat, il fallait quatre juments, une brebis et une chèvre (Girčenko, 14)

6. Alcool distillé à partir du lait fermenté, complété hors saison par de l'alcool-de-grain, adoptê avec l'agriculture.

7. Les accessoires sont fumigés à 1'aide d'un morceau incandescent d'écorce d'épicea ; les assistants doivent, au sortir de la yourte, enjamber un feu aromatisé de serpolet ou de bruyère. A ce feu chaque maître de maison purifie 1a "part" xubi (a1cool et récipients) qu'il apportera sur la montagne (cf. Hamayon, 1977).

8. S'il n'y a pas assez de chevaux pour tout le monde, on monte à deux sur le même; la tradition semble interdire de s'y rendre à pied.

9. Sans entrer dans la complexe réalité clanique, on peut indiquer qu'en moyenne 5 à 8 patrilignages vivent dans un même campement, comptant de 25 à 40 maisons. Le clan (jahon, "os") regroupe 3 ou 4 de ces campements. Une famille nucléaire -- augmentée d'éventuels isolés -occupe une maison. Au tajlgan chaque patrilignage (urag) a sa branche de bouleau signalétique (tüürge) et son chaudron. 
Avant d'être abattus, les animaux ont droit eux aussi à goûter au lait fermentê ou à l'alcool. Un chaman s'approche de chacun d'entre eux, lorsqu'on 1'abat, pour inviter son âme à s'en aller dans la direction fixée, vers laquelle sa tête dẻjà est tournẻe. Après dépeçage, la chair de chaque animal est mise à cuire dans un chaudron à part. Longue est la cuisson, laissant aux vieux tout loisir de boire, aux jeunes tout loisir de danser, jouer, lutter, courir. La viande qui se détache elle-même des os (avant cuisson selon Sanžeev, après selon d'autres auteurs), appelée dalanga, est destinée à être rapportée à la maison. Celle qui reste sur les os (šuladahan, terme formé sur la racine "râcler") est soigneusement enlevée, puis transportée dans les auges jusqu'au tüürge commun, ensemble de bouleaux signalétiques au rôle d'autel. Quelques menus morceaux en sont jetés au feu commun, dans un crépitement couvert par les invocations, et le reste est obligatoirement consonmé sur place.

Les os sont incinérés sur la ou les pierres claniques (parfois de vraies petites constructions, šeree) sans mélange, pour chaque animal séparément, comme pour la viande. Quant aux dépouilies, conservant d'un seul tenant la tête, 1'appareil respiratoire, les poumons et le coeur (10), avec de surcroît de petites branches de bouleau dans les narines et de l'écorce d'épicéa sur le front, elles sont enfilées sur des perches dans la direction de la zone surnaturelle où résident les dédicataires.

Arrosé d'alcool, le festin bat son plein jusque fort avant dans la nuit, agrémenté de jeux et danses rituels, au rang desquels figurent les pantomimes du chaman, incarnant divers esprits. On reviendra chez soi, chacun à son heure, avec la viande dalanga.

Le plan de ce rite compte pour épisodes marquants : la purification, le départ vers l'espace sacrificiel et sa délimitation, 1'aspersion accompagnant l'invocation aux ancêtres, le traitement de I'âme, de la chair et de I'os de l'animal sacrifié, le festin à interludes dramatiques. En tant que rituel sacrificiel, le tajlgan se présente comme collectif, régulier, préventif ou propitiatoire, et structurellement liê à l'institution clanique.

b. xereg.

Ce nom, traduisible par "affaire", porte une nuance d'utilité, voire de nécessité. Affaire privêe, ce type de rite peut toutefois connâtre une large participation. Son motif peut être aussi bien le

10. L'ensemble, appelé huuraj ou hülde selon les régions, n'a pas de traduction française appropriee. Le terme de fressure, en effet, inclut le foie et la rate, mais ne recouvre pas la tête. 
rappel de l'âme d'une femme déprimêe par la discorde conjugale, qu'une demande d'enfant pour un couple stêrile, la guérison d'une calamiteuse maladie frappant la famille ou le bétail, la solution d'un conflit provoqué par la colonisation, la récupération d'une partie égarée du troupeau, etc. Cependant la majorité des."affaires" est accomplie pour affermir ou recouvrer la santé des enfants. Une affaire-type se déroule grossièrement ainsi :

Les accessoires rituels sont fumigês sous la yourte devant la famille assise par le chaman, dont le concours est ici expressément requis. Au sortir de la yourte, le chaman asperge et invoque. Le rituel proprement dit se déroule dehors, à quelques mètres devant la yourte (11). L'autel, fait d'une branche de bouleau.(tü̈̈rge), marque le coeur de l'espace sacrificiel : au nord un chaudron, entre les deux une pièce de feutre couverte des récipients sacrificiels. Une nouvelle aspersion précède 1 'abattage de la ou des victimes. C'est alors que le chaman chamanise : en sueur et secoué de tremblements, il mime son voyage à la rencontre de 1 'esprit dédicataire; le récit des pêripéties de sa vie terrestre et de ses apparitions posthumes, tel est le prélude à l'invite faite à l'esprit d'accepter ce qu'on lui offre; peu après, le chaman s'enquerrera si la victime est agréée ou non. En cas de refus, le sacrifice devra être recommencé. C'est également la divination qui avait permis au chaman de déceler le responsable du désordre, déterminé le sacrifice propre à y remédier, l'espèce et la couleur de la victime.

Au retour dans la yourte, le chaman fera une dernière aspersion d'alcool.

En somme, tout en préservant les grandes lignes de son déroulement, 1'"affaire" s'oppose au sacrifice clanique par son caractère privé (mo$t$ if, espace, assistance), occasionnel, curatif ou expiatoire, et par son lien fondamenta1 avec 1 'activité chamanique.

c. dolig (doljoo).

Plus qu'un type rituel à proprement parler, le dolig, "rançon", apparaît comme un cas particulier d'"affaire". Son nom même êvoque clairement le sens du rite. Formellement analogue à 1'"affaire", 1a "rançon" s'en distingue dès 1 'abord par la gravité de son propos (c'est la vie d'un agonisant qui est en jeu), par la tension de son atmosphère,

11. Prescription rituelle qui, comme le remarquent plusieurs auteurs (par exemple Manžigeev, 175), risque d'être fort préjudiciable au malade, si l'on est en hiver. 
et par la minutie anxieuse mise par le chaman au choix divinatoire de la victime, dont tout, sexe, âge, couleur, doit être approprié. C'est le rôle assigné à la victime qui fait l'originalité de la "rançon".

Après les préparatifs habituels, on fait entrer l'animal dans la yourte auprès du malade, qui doit lui cracher dessus trois fois (12). Puis, on 1 'emmène dehors pour 1 'immoler et le préparer comme à l'accoutumée, à l'exception de sa pseudo-fressure, huuraj (cf. note 10). Celle-ci est apportée, agrémentée d'un peu de bouillon, au malade qui en inhale la vapeur, et est conservée jusqu'à sa guérison (Xangalov, I, 512 et II, 202-212).

Le substitut proposé en rançon pouvait être jadis un humain, dans un temps encore accessible à la mémoire de I'homme, et la mythologie en compte plusieurs exemples. Le traitement rêservé au substitut humain varie : celui-ci est tantôt vraiment sacrifié comme 1'animal, mais incinéré (à 1'exception de son huuraj), tantôt seulement proposé (sous la forme de son âme) par le chaman à l'esprit avide -- ce qui suffit à 1 'entraîner dans la maladie et la mort.

Ce second traitement symbolique est le plus fréquent dans les récits. I1 est le fait d'un chaman noir et puissant, qui effectue en accord avec le malade ou le chef de famille le choix de la victime offerte en "rançon". Ce choix porte d'ordinaire sur les proches du malade, surtout sur' ceux qui sont encore dans I'enfance. L'âme valant pour la personne, étant son double et conditionnant sa santé et, à la longue, sa vie, il suffit au chaman de ravir une âme pour l'offrir en rançon de l'âme du malade, détenue ou tourmentée par quelque esprit. Aussi lorsqu'un chaman vient officier pour un grand malade, entretient-on, dans les yourtes du voisinage, du feu toute la nuit pour s'empêcher de dormir et prévenir ainsi toute fugue de l'âme. En effet, l'âme profite du sommeil pour faire de petits tours hors du corps, et se trouve alors vulnérable. Si elle est interceptée, la mort du corps s'ensuivra fatalement.

Le chaman doit, ici plus que jamais, vérifier 1 'acceptation du substitut par le dédicataire. Celle-ci, du reste, ne procure au malade racheté qu'un sursis provisoire : son âme réintégrée risque toujours une nouvelle capture. Cette restriction participe d'un principe fondamental du chamanisme bouriate : borné à l'éloignement des désordres, le pouvoir chamanique n'assure à la société que le maintien du statu quo de son existence dans le monde, sans prêtendre lui faire retrouver un éventuel paradis, initial, ni lui laisser entrevoir quelque issue que ce soit. 
La "rançon" rejoint l'"affaire" du côté du privé et du curatif, ainsi que dans l'étroite dépendance à l'égard du chaman, mais à son encontre, implique éventuellement un manque apparent de solidarité entre humains.

Un bilan provisoire montre que seul le tajlgon, la "cour faite aux esprits" meut toute la collectivité. Il se situe en tant qu'acte rituel du côté de la norme, du maintien de l'ordre implicite, alors que les deux autres rites assurent la restauration de cet ordre, par éloignement des désordres réels, au prix parfois de transgression de règles sociales ou symboliques.

\section{SACRIEIANTS (BENEFICIAIRES)}

Sont qualifiés ici de sacrifiants ceux qui fournissent la victime, organisent le sacrifice et en règlent l'exécution, soit, dans le cas du sacrifice clanique, tous les membres mâles adultes du clan et en particulier les â̂nés, et, dans le cas de 1""affaire" comme dans celui de la "rançon", le chef de la famille touchée par le désordre, que celui-ci soit matériel, biologique ou psychologique. Il n'y a donc pas de sacrifiant professionnel, officiant au service d'autrui. Sacrifie qui recherche un bénéfice, pour lui-même ou ce qui dépend de lui : famille, troupeau, territoire, le rôle du maître des cérémonies échouant en gênéral à un aîné. Tout ceci amène à constater une identification relative entre sacrifiant et bénéficiaire (direct ou indirect).

I1 arrive que des lignages ou même des campements entiers soient acculés à l'endettement et, de 1à, à l'asservissement, pour avoir épuisé leurs ressources en sacrifices, à la suite de catastrophes réitérées, ou par excès de précautions, ou encore par soumission au zèle

12. Cracher équivaut simplement ici à tenter de transférer son mal à l'animal. 
de quelque chaman peu désintéressé.

Les faces sociales et religieuses du sacrifice clanique se mêlent étroitement : grand rassemblement saisonnier des membres épars d'un clan, il sauvegarde sa cohésion, sa solidarité, réaffirme ses prérogatives territoriales. En ce sens, il apparâtt comme une communion, au large déploiement synchronique et diachronique, à 1'allure de grande fête. Il entretient aussi la sécurité spirituelle du groupe, placée sous la garde des ancêtres-maîtres des lieux qui, moyennant la rétribution que constitue pour eux le sacrifice, garantissent aussi, symboliquement, sa sécurité physique et matérielle : fécondité des hommes, des troupeaux, clémence météorologique, protection de principe contre toute catastrophe de type connu ou inconnu. Ce qu'on attend concrètement d'eux, cette prière le dira mieux :

Au loup qui traîne sa queue

Emoussez les dents,

$\mathrm{Au}$ voleur qui traîne sa longue perche-lasso

Raccourcissez 1a perche-1asso !

Repoussez ce qui a du noir, ce qui a du gris,

Amenez ce qui est bon, ce qui est beau !

Devant nous soyez la vigile,

Derrière nous, soyez 1 'ombre !

Ne nous laissez pas mordre par les chiens,

Empêchez la nuisance des méchants,

-.

Rendez-nous riches en chevaux,

Souhaitez-nous prolifiques en petits-fils,

Nous vous offrons de 1 'alcool-de-lait

Abondant comme la grêle céleste !

...

Du haut du ciel donnez-nous chance et fortune,

Du large de la terre donnez-nous chance et richesse !

Parents d'origine dont nous sortîmes,

Parents d'origine dont nous naquîmes,

...

Dîtes ce que nous n'avons point dit,

Pensez ce que nous n'avons point pensé,

Comprenez ce que nous n'avons point compris ! (Baldaev, 1975,174175).

Invocations, aspersions, divination incombent aux plus âgés ou aux plus doués. Si un chaman entre en scène, c'est seulement en tant que membre du clan particulièrement rompu à ce genre de tâche. Immoler les victimes ne revient ni aux vieux ni aux chamans (13), mais à qui excelle

13. La seule occasion où le chaman doive lui-même immoler 1'anima1 est son ablution sanglante de "prise d'habit". 
dans le maniement du couteau, possède le savoir-faire et la force requise d'un sacrificateur. Son rôle, limité à l'abattage, est concret, tandis que celui dévolu aux invocateurs a aussi une part symbolique : chargés de rendre le sacrifice efficace, ils doivent respecter les règles liturgiques, prësenter la victime de manière à contraindre les dédicataires à 1 'accepter et à agir en conséquence. Ils y ont tout intêrêt : eux-mêmes, leurs familles, troupeaux, pâturages en sont les bénéficiaires prêsumés.

Dans 1'"affaire", l'assistance, quoique moins nombreuse, ne se 1imite pas à la famille du sacrifiant. A l'idée que la présence humaine exerce une pression positive sur le chaman et sur l'esprit fauteur de trouble s'ajoutent une insatiable curiosité (14) et l'attente du festin. Si le chaman est ici indispensable, c'est autant pour choisir l'animal et l'envoyer à bon port que pour obtenir, si j'ose dire, l'accusé de réception. En effet, des esprits autres que celui qui est invoqué -- surtout ceux de chamans défunts de clans ennemis, toujours aux aguêts --, pourraient intercepter l'offrande. Mais le plus grand risque est de commettre une erreur liturgique : les esprits n'en tolèrent aucune; le chaman tient-il a l'envers I'écorce d'épicéa (cf. note 7), place-t-il à droite la pouliche blanche et à gauche le bélier blanc au lieu de l'inverse, se trompe-t-il de couleur, que la victime est refusée et que tout est à recommencer. "Ainsi chante un chaman, agitant un morceau d'épicéa plongé dans le sang de 1 'animal :

Substitut pour une vie,

Cadeau pour un corps, Fêlicité approche-toi, Calamité éloigne-toi !

Si l'invocation n'est pas comme il faut, Que la faute en retombe sur le chaman! Si la victime n'est pas bien préparée, Que la faute en retombe sur qui l'a préparée!

Si tout est comme il faut

Et que les esprits ne sont pas miséricordieux, Que la faute leur retombe dessus !... Plus légère que la plume, plus rapide que la flèche, Calamité écroule-toi ! (15)."

14. Suscitée par la fonction dramatique remplie par toute activité chamanique.

15. "Puis, poursuit Ordynskij, 41-42, le chaman entre en transe, incorpore l'esprit cause du mal, crie, gémit, hurle, gesticule. On le retient ; il s'endort environ une demi-heure, pendant laquelle il rencontre les esprits. Au réveil, il tremble, l'oeil lointain, fiévreux ; on lui donne une tasse d'alcool, et il chante une nouvelle supplique." 
C'est le chaman lui-même qui, par divination, décèle ses erreurs -- comme le chaman Laxtan qui mourut d'avoir prêsenté en sacrifice un cheval noir au lieu d'un blanc (Sanžeev, 607). Parce qu'un support de dépouille était planté à l'envers, le clan Jangut fut réduit à son dixième, les rescapés devant émigrer (Manžigeev, 23-24). D'une faute liturgique, le malade ne sera pas plus mal en point, la sécheresse ne sera pas plus épouvantable, mais un animal aura été tué pour rien. En effet, le résultat ne découle pas de l'application automatique de règles préexistantes. Une part des décisions est élaborée au fur et à mesure par la divination du chaman, qui s'évertue à choisir une victime exempte de défauts susceptibles de déplaire au dédicataire. Si les humains mâtrisent la manipulation du concret et détiennent l'initiative de la conduite du sacrifice, ils ne décident pas de son exécution et tremblent sans cesse devant les facéties qu'ils prêtent à la surnature (16). Qu'une part de l'issue d'un acte soit laissêe aux caprices du symbolique, et 1 'angoisse est là.

16. Par convention, est appelé "surnature" l'ensemble des êtres symboliques peuplant I'univers des Bouriates; cela ne préjuge nullement que ces êtres fassent l'objet d'une croyance de nature différente de celle qui porte sur les êtres réels. Le terme de surnature recouvre plusieurs catégories d'êtres, qu'aucun terme bouriate ne désigne globalement :

--tengeri, ciel atmosphérique et esprit qui y réside et qui mâ̂trise le phénomène correspondant (grêle, orage, etc.) ; les 55 tengeri blancs de l'Ouest, dêtenant le pouvoir légitime en tant qu'aînés, sont de ce fait plutôt bénéfiques ; les 44 tengeri de 1 'Est, noirs et cadets, contraints à la subversion, sont à l'origine de tous les fléaux.

-- Ancêtres et héros, maîtres de terres et des eaux, et simultanément mâ̂tres des diverses activités humaines (chasse, forge, êlevage des chevaux, etc.) et de la santé physique et mentale des hommes et des animaux domestiques. Il en est d'échelle nationale et d'échelle régionale. Alors que les ancêtres se fondent dans un ensemble anonyme, les héros, mythiques ou historiques (souvent de grands chamans) sont individualisés. Aux premiers sont dêdiés les tajigan d'ouverture et de clôture de l'estivage, aux seconds les tajlgan occasionnels.

-- zajaan, 1itt. "destin", esprits des trépassês, morts de mort non naturelle, cf. plus en détail le chap. IV, Dédicatasires. Perdant avec le temps de leur nuisance, une part d'entre eux a tendance à se confondre avec les héros (cf. ci-dessus).

-- Foule des esprits issus des âmes de morts ordinaires et même de morts irréguliers n'ayant pas atteint le degré de zajaan ; leur influence est mineure; ils ne reçoivent pas de culte.

La méfiance envers les êtres de la surnature provient essentiellement de ce que ceux-ci, anciens hommes, gardent dans $1^{\prime}$ au-delà leurs défauts et leurs qualités d'hommes. 
La "rançon" rêunit une assistance plus restreinte; la clandestinité est de règle lorsque le substitut est humain : dans ce cas deux personnes seulement sont indispensables, le bénéficiaire et le chaman, la victime n'entrant en scène que symboliquement à la suite du rapt de son âme par le chaman. La "rançon" offre le seul exemple d'identification entre la victime -- qu'elle soit animal réel ou humain symbolique -- et le bênéficiaire, l'un étant le substitut avoué de l'autre. Une telle substitution n'est possible qu'au sein de la parenté du bénéficaire si la victime est une âme humaine, au sein de son cheptel si la victime est un animal -- comme si le substitut devait provenir, en tout état de cause, du patrimoine du groupe en hommes ou en bêtes, comme si aussi une âme de bête valait bien une âme d'homne au goût đu dédicataire (17). Rien de semblable n'était perceptible dans les deux premiers rites, où le traitement des victimes excluait même 1'éventualité d'une identification avec les bénéficiaires (18).

$$
\text { III. SACRIFIES }
$$

a. Viatimes animales.

Le gibier est par principe exclu du sacrifice. Prélevé dans la surnature, il ne saurait y être retourné, dédié. C'est donc dans son cheptel ou par achat que le sacrifiant s'approvisionne en victimes. En Cisbaïkalie, 1'élevage, de type extensif, porte de façon conjointe et complémentaire sur quatre espèces (chevaux, bovins, ovins, chèvres),

17. L'efficacité du sacrifice repose sur des mécanismes symboliques, dont celui de la substitution (cf. Lévi-Strauss, Le totémisme aujourd'hui, Paris, PUF, 1962, p. 8 ). Celle-ci toutefois ne peut s'exercer qu'à 1 'intérieur de certaines limites, concordant avec les principes taxinomiques (cf. p. suiv.).

18. En revanche, une identification implicite se fait jour dans deux croyances apparement contradictoires rapportées par Xangalov (I, 396 et II, 210) : "le cheval qui meurt dispense son mâ̂tre de mourir", et "de 1 a mort du cheval, s'ensuivra tôt ou tard la mort du maître", correspondant chacune à un contexte différent. 
auxquelles s'ajoutent, en Transbaïkalie et en Mongolie, les chameaux. La taxinomie populaire attribue aux chevaux et aux moutons des museaux chauds, aux autres des museaux froids. En principe, seuls les museaux chauds sont sacrifiables, mais on constate que plusieurs rites imposent d'immoler des chèvres. Certes, on ne surprendra jamais un Bouriate à sacrifier un bovin, et on le stupéfierait en lui suggérant d'immoler un chien : tout n'est pas bon à servir de substitut à tout (cf. note 17).

Les victimes ne doivent être ni castrées, ni marquées. Leur couleur est impérativement prescrite (la couleur pie est exclue) : blanche ou du moins claire pour les dédicataires relevant de la moitié blanche du ciel (cf. note 16), selon le principe d'assortiment de la couleur des attributs du maître à celle de celui-ci. Les règles, assez lâches pour le tajlgan à 1'exception du sexe (femelles exigées), sont plus précises pour les "affaires" et surtout pour les "rançons", comme en tẻmoignent les nombreux exemples d'échec pour cause d'erreur sur la couleur ou la disposition des animaux. On trouve spécifiés une jument jaune rousse pour les funérailles d'un père, un poulain, un bélier et un bouc pour 1e maître des eaux, un chevreau blanc à châtoiement jaune pour tel rite de petite enfance masculine... L'état actuel des recherches ne permet pas d'établir les bases du choix, ni l'éventuelle correspondance entre le type de victime d'une part, le type de rite, de bénéficiaire, de dédicataire ou de désordre à réparer. On peut tout juste pressentir que1ques orientations. Obscures restent les raisons d'immoler des boucs et des chèvres qu'en principe leur museau froid rend inaptes au sacrifice. Les rituels qui les veulent pour victimes mettent en gënéral en scène des êtres en état d'infériorité sociale, provisoire ou permanent : -- d'une part, les jeunes enfants des deux sexes, ainsi que le chaman, assimilé à un nouveau-né lors de sa "prise d'habit" ;

-- d'autre part, les femmes; les petites filles lors de I'ablution de naissance $\left(b u d \gamma_{j} \alpha\right.$ ), rite qui impose le sacrifice d'un bëlier pour les garçons. C'est aux femmes qu'est donné le chevreau sacrifié lors de la "prise d'habit" du chaman. Une aîeule mythique, mère de trois ancêtres fondateurs issus du lac Bažkal, serait apparue sous forme de chèvre de façon que, par la suite, on lui en offrît (Xangalov, III, 104). Dans la hiérarchie culinaire, le plat de chèvre est tenu pour inférieur (19). Victime inférieure pour bénéficiaires inférieurs ou en état d'infériorité, tel serait le statut des caprins. 
Pour finir le recensement des victimes animales, il faut citer que1ques mentions isolées de poisson offert à la Lotte Pie, maîtresse du Baîkal et ancêtre des Exirit, de gelinottes et de coqs de bruyère à l'Aigle mâ̂tre de 1'île d'01'xon, premier chaman (Xangalov, passim).

b. Victimes humaines.

Ne sont clairement définies comme humaines que les victimes immolées à l'emblème militaire d'un groupe, le hülde (mongol süld ; cf. Burdukov, Žukovskaja). Queue de yak ou de cheval, cet emblème garantit la prospéritê du groupe tant qu'il est intact. On 1'abreuve du sang d'ennemis prisonniers pour donner le goût d'en boire à nouveau, autrement dit l'ardeur à la guerre, et finalement la victoire aux guerriers qui s'en réclament. C'est le seul cas où le sang coule (20). Quant au choix de 1a "rançon" humaine, I'appartenance au même patrimoine est, comme il a êté dit ci-dessus, la principale prescription.

\section{c. Traitement des victimes animates.}

C'est 1 'examen du traitement réservé aux victimes qui peut, à cette étape, nourrir quelque réflexion sur leur position symbolique.

L'abattage, qu'il soit rituel ou profane, suit toujours le même processus : I'animal est d'abord fumigé et régalé de laitage fermentê; puis, les pattes liées deux par deux, il est renversé sur le dos et couché sur un feutre blanc. D'un coup de couteau éclair, on lui fend la poitrine, et d'une main leste on lui arrache 1'aorte. Quelques secondes, et la mort est là, sans une goutte de sang sur le feutre blanc. Le sang est recueilli par pression sur l'aorte, dans un récipient spécial : les femmes en feront du boudin.

19. La chèvre, cuite dans sa peau à l'aide de pierres portées au rouge, est le seul plat de viande frais à être consommé totalement en plein air. C'est le plat de pique-nique par excellence, et un des meilleurs cadeaux alimentaires offerts à qui part en voyage, sans qu'il y ait, dans ces cas, la moindre nuance dépréciative.

20. La perspective d'avoir son sang qui coule fait frémir les guerriers de l'Empire faits prisonniers (cf. Jamuqa, dans L'Histoire Secrète des Mongols) ; c'est semble-t-il un présage funeste pour leur descendance tout autant qu'une perte de leur pouvoir symbolique au-delà de la mort. De même le chaman condamné par le jugement populaire doit être battu jusqu'au sang pour perdre tout son pouvoir et sa nuisance. 
Si le sang animal (21) ne semble pas intéresser les esprits, celui de l'home leur plaît si l'on en juge par les invocations d'esprits avides et repus de chair et de sang humains (Xangalov, II, 203). La viande sacrificielle ne semble pas non plus être, en premier lieu, un aliment d'esprit; toutefois certains rites interdisent tout sacrifice ou toute distillation sous prétexte que le dédicataire ne mange pas de viande ou ne boit pas d'alcool. C'est à l'intention de l'esprit-maître du feu que quelques menus morceaux sont jetés dans les flammes, pour le nourrir. La quasi-totalitê de la viande est ouvertement et explicitement consommée par les humains selon des manières qui tiennent de la communion.

Les os, quant à eux, ne doivent jamais être fracturés ni égratignés lors du dépeçage et du râclage de la viande : le moindre dommage empêcherait la victime d'être agréée. En effet, la croyance fait de 1 'intégrité des os la condition de la rêincarnation sur terre et de la survie symbolique dans le troupeau surnaturel. Le squelette est, en quelque sorte, l'armature essentielle de l'individu, associè à une âme qui est son double, os par os et globalement. Si les os sont incinérés, ce n'est pas en tant que restes qui doivent disparaître, mais pour que soit symboliquement transféré au troupeau surnaturel le squelette : à partir des cendres, 1'animal pourra y être reconstitué (22). L'incinération, qui aboutit apparement à une destruction totale, est en fait le type bouriate de dénaturation qui exclut la réincarnation sur terre. Car seule une dénaturation définitive rend le transfert irréversible.

"Prenant appui sur tes quatre sabots noirs, entre $[-z-y]$

Grinçant de tes quarante dents blanches, entre [-z-y]

dit le chaman -- selon. Sanžeev (p. 579) -- à l'"âme-sosie" du squelette à reconstituer.

On retrouve dans les funérailles le même principe de dénaturation

21. Les anciens Mongols buvaient le sang frais de leurs chevaux, révèlent les voyageurs du Moyen Âge (Rockhil1, xv-xvi). On se sert chez les Bouriates (Sanžeev, légende p. 934) du sang provenant du coeur de $I^{\prime}$ animal sacrificiel pour peindre les figurations d'esprits.

22. De même, les héros de l'épopée sont reconstitués à partir de leurs cendres, puis ranimés. 
destinêe à rendre 1e trêpas irréversible : 1'âme s'échappe pendant 1a crémation (23) du cadavre, et ne trouve plus ensuite d'enveloppe où s'incorporer. En revanche, on se garde de toucher au cadavre de 1 'enfant en bas âge : un enfant doit renâtre dans la famille. Les seuls morts dont l'âme rôde malencontreusement sont ceux que l'irrégularité de leur vie et de leur mort a empêchês d'être incinérés comme tout le monde.

Le sort réservé à la dépouille sacrificielle procède de la même intention d'augmenter le troupeau surnaturel d'une unitê, prêlevée sur le troupeau terrestre. Tous les détails rituels le confirment :

- le soin de laisser d'un seul tenant le huuraj (cf. note 10) qui est le support organique du souffle vital, amin, lequel est la seule instance indispensable à la vie, et donc le gage de la re-vie symbolique de l'animal (qu'il soit laissé dans la dépouille ou incinéré avec les os, comme cela se fait chez les Bouriates Alar').

-- le soin de préserver entière la peau, préservant ainsi 1 'apparence de 1 'animal vivant;

- 1 'orientation de la dépouille sur une perche dressée

obliquement vers son futur lieu de résidence. (Elle y est laissée jusqu'à ce que les rapaces aient achevé de la dévorer).

- les textes d'invocation: "le gros et gras cheval, à vous nous 1'offrons" (Baldaev, 1975, 171).

L'intention des humains est d'offrir à la surnature non pas la chair, d'usage éphêmère, d'un animal, mais cet animal lui-même, à 1'intégritê soigneusement préservée -- ce dont témoignait déjà le souci de ne pas mélanger les os et les chairs des diverses victimes (24). N'estce pas, somme toute, d'une insigne habileté que d'offrir un animal entier symboliquement vivant, sans se priver de sa viande bien réelle.

Si la victime ne doit pas être marquée, c'est que tout signe

23. Très répandue, 1 'incinération n'êtait pourtant pas gênérale ; dans certaines régions, particulièrement en Alar', le cadavre était mis en cercueil et enterré, face contre le sol, de façon à empêcher 1 'ame de retourner sur terre. La coutume lamaíque engage à laisser le cadavre à même le sol : plus vite il est dévoré par chiens et rapaces, et mieux ce sera pour lui-même et pour sa parenté vivante.

24. A ce propos, une remarque sur 1 'os et la chair, célèbre couple des parentés asiatiques. Comme toujours, la valeur positive revient à 1 'os, avec la modalité durable, mais virtuelle, en face de celle; éphémère mais réelle, de la chair. (Pour la comparaison avec d'autres domaines, où intervient ce couple, cf. Hamayon, 1975, p. 117). 
d'appartenance entraverait le changement de propriétaire. Que les victimes des principaux tajıgan d'été soient plutôt femelles n'est pas un hasard ni une commodité : on est aussi friand au-delà qu'ici-bas de laitages fermentés et distillés. Ainsi les hommes procurent-ils à la surnature abondance et prospérité.

Indubitable, ce double facteur de productivité n'est toutefois qu'un parmi d'autres à intervenir dans la sélection des victimes. Sinon, comment expliquer qu'aucun bovin ne soit jamais immolé ? Plus qu'à son museau froid, c'est au rôle d'ancêtre fondateur de l'ethnie, joué par le Taureau Bleu, fils des cieux de l'Ouest, que l'espèce bovine doit d'être préservée. Comme on le verra plus loin, le destin du taureau est la consécration, qui lui vaut aussi d'être tué, mais pas sacrifié. La vache, quant à elle, est en dehors de tout circuit rituel. On raconte l'histoire des deux jeunes filles de Xori qui moururent de ce que leur marâtre, fêlonne et férue de lamaisme, fit sacrifier une vache noire au lieu du cheval blanc prescrit par le chaman. En outre, il est des bêtes qui, non contentes de produire et de se reproduire, sont fastes : pour rien au monde on ne saurait s'en défaire, leur "grâce" ( $x$ ǐs̆ig) risquant alors de passer à 1 'acheteur ou au dêdicataire.

I1 n'y a, dans ce transfert d'une unité d'un troupeau à un autre, rien qui ressemble à une identification de la victime avec l'un quelconque des autres partenaires du sacrifice : tout au plus se souciet-on d'assortir la couleur du troupeau à celle du ciel de résidence. I1 n'y a pas lieu de revenir ici sur l'identification du sacrifié avec le bénéficiaire dans le cas de la "rançon", puisqu'elle a déjà été relevée à propos du bénéficiaire. On peut toutefois pénétrer maintenant plus avant dans les motifs du choix du substitut. Si parmi tous les substituts possibles que compte le patrimoine du groupe, on préfère offrir en "rançon" les enfants (25), c'est que ceux-ci, mourant avant 1 'âge d'être introduits dans la société, conservent un droit de vie sur terre -- ce qui n'est pas donné aux adultes qui, eux, ont vécu

25. Et les jeunes animaux ? Il est souvent prêcisé "poulain de moins d'un an", par exemple, mais il n'est pas explicite que le jeune animal mort revivra sur terre. 
leur temps de vie (26).

$$
I V \cdot \quad D E D I C A T A I R E S
$$

Même si les cieux de 1'Ouest et de 1'Est (cf. note 16) sont nommés les premiers dans la plupart des invocations, ce n'est pas à eux que sont envoyés les animaux des tajlgan, mais à leurs descendants plus ou moins immédiats, qui se confondent souvent avec les ancêtres mythiques des humains. Accompli obligatoirement sur 1a pierre de résidence des fondateurs claniques, le tajlgan est l'élément le plus spectaculaire des relations contractuelles qu'entretient une communauté de vivants avec la communaté de ses morts.

$C^{\prime}$ 'est aussi à d'anciens morts que sont dédiées, dans la quasitotalité des cas, les victimes des "affaires" et des "rançons". Loin de constituer de dignes lignées d'ancêtres, ce sont des morts qui se prêsentent plutôt comme d'indignes anti-ancêtres; on les regroupe sous le nom de zajaan, "destin", "destinëe". Ils sont les frustrês de la surnature bouriate : frustrês d'avoir trop brièvement vécus, d'avoir été mal aimés et mal intégrés par la sociêté, et de n'avoir pas eu de funérailles normales, par suite de leur mort anormale; pour n'avoir pu être correctement transférés dans 1'au-delà, ils errent entre les mondes en mal d'insertion, en quête de toute occasion de vengeance. on connaît de ces âmes en peine qui ruinent les gens de la terre par leurs exigences en sacrifices, exigences qu'elles manifestent par les troubles les plus divers : elles les hallucinent en pleine nuit dans la forêt, les font tomber de cheval, les rendent somnambules, portent atteinte à leur santé mentale (plus rarement physique), nuisent à leurs enfants ; peu après elles auront droit à leur chaudron d'alcool et à leur victime favorite. En fait, d'entre tous les occupants dè la surnature, les zajaan sont ceux dont la présence aux vivants est la plus intimement ressentie, la plus quotidienne, et qui

26. La réincarnation des enfants morts en bas âge est impliquée par le rituel funéraire, le rituel de naissance de l'enfant suivant, etc., et reconnue explicitement. 
partagent avec eux la responsabilité des choses de ce monde, de par leurs arbitraires et fatales sautes d'humeur, et leur désir de vengeance.

$\begin{array}{cccccc}V \cdot \quad U N \quad G E S T E & A \quad L I E G A R D \\ D E & P A R T E N A I R E S & S Y M B O L I Q U E S\end{array}$

Les relations avec les morts calquent celles que 1 'on a dans $1 \mathrm{a}$ cérémonie d'hospitalité avec des partenaires terrestres. En effet, sacrifice et hospitalité semblent obéir au même modèle précis de déroulement : même prélude d'alcool-de-lait aspergé avant d'être absorbé, même procédé d'abattage de 1 'animal à consommer, même sort de boudin réservé au sang, même séparation hiẻrarchique entre viande molle et viande attachée à l'os, même soụci de ne pas endommager les os pour favoriser la réincarnation au-delà ou ici-bas ; même obligation de consommer la chèvre entièrement sur place; même disposition des rëcipients sur 1 'autel et sur la table, qui portent le même nom, Seree ; même disposition, par rang d'âge (ou de niveau gênéalogique), des participants.

Hospitalité et sacrifice valorisent de façon analogue et complēmentaire un même type de circuit de prestations, une même orientation du comportement courtisan : le partenaire qui honore le plus, dans ce milieu nomade, est celui qui se déplace. Marque de courtoisie, sa visite est en même temps la présentation de cadeaux de nourriture et de valeurs (fourrures, tissus); elle est surtout une invite à la conclusion d'un contrat qui réclame du demandeur une attitude de déférence. Aussi celui qui reçoit la visite s'efforce-t-il, pour réduire sa position d'obligé, de surenchérir, en déployant tous les trêsors de son hospitalité à l'intention de son visiteur.

Entre vivants et morts d'un clan, la répartition des rôles n'est pas la même qu'entre partenaires d'hospitalité. D'une part les ancêtres n'ont rien à solliciter des vivants, aussi ne sauraient-ils sans déchoir rendre visite à leurs descendants chez ces derniers. D'autre part, détenteurs des droits et du bonheur des vivants, ils attendent d'être dûment rétribués pour octroyer des bienfaits, et font la sourde 
oreille s'ils s'estiment insuffisamment honorês. A la passivité de leur attitude répond le double hommage des vivants, qui se déplacent jusqu'à leur lieu de résidence à flanc de montagne et les y régalent du meilleur de leur chaudron. Ici, un partenaire est doublement récepteur, 1 'autre doublement donneur.

En revanche, c'est comme un hôte de passage et devant sa propre yourte que le maître de maison traite les esprits des frustrés en rêponse à leur funeste visite. On ne prend pas d'initiative à leur égard, et lorsqu'ils se manifestent, on cherche à les neutraliser, à les empêcher de nuire, alors qu'on s'évertue à rendre visite aux ancêtres pour les propitier. Avec ces derniers, le sacrifice est un contrat positif de bons offices, avec les autres un pacte négatif de cessation des hostilités.

En outre, sacrifice et hospitalité, 1 'un envers les morts, 1'autre envers les vivants, créent des situations extrêmement contraignantes pour qui en est gratifié, car celui-ci est mis dans l'obligation d'accepter les présents et le festin offerts, et par là, le contrat proposé. Le parallélisme entre les deux rites incite à prêter à la démarche humaine dans le sacrifice une note nettement coercitive.

Enfin, comparables par l'attitude envers les partenaires aux relations rituelles entre humains, les trois rituels de sacrifice empruntent leur nom même à la terminologie des relations : "faire la cour" marque un rapport d'inférieur à supérieur, tandis que 1'"affaire" et la présentation d'une "rançon", strictement contractuelles, mettent les partenaires sur un pied d'égalitê. Le premier rite assure ordre, permanence et prospérité, tandis que les deux autres effectuent un troc d'âmes pour rétablir le statu quo entre la société et ses frustrés trépassês. I1 faut toutefois faire mentalement craquer les limites de ce schêma un peu rigide pour qu'il accueille d'une part les "affaires" faites aux ancêtres en cas de transgression d'interdits, et les tajzgan préventifs adressés à certains zajaan, esprits de frustrés, qui n'arrêtent pas de sévir après leur mort, mais deviennent, avec le temps, de moins en moins néfastes, et même dispensateurs de bienfaits. 


$$
V I . \quad U N E \quad M I S E \quad A \quad M O R T
$$

Même non sacrificielle, toute mise à mort présente un caractère rituel, qu'il s'agisse :

-- de la mise à mort du bétail, matériellement identique à celle d'une victime sacrificielle, effectuée pour donner l'hospitalité ou pour constituer à l'autome les réserves de viande, car il n'est jamais anodin pour un pasteur d'entamer son cheptel.

-- de celle du gibier, dont les âmes proviennent de 1a surnature. Tuer un ours ou les petits carnassiers, dont les fourrures entrent dans 1 'arsenal chamanique, impose un rituel délicat. Funeste est de tuer par inadvertance un volatile interdit comme le cygne, ancêtre mythique.

-- de celle des condamnés divers, car il en émanera de vengeresses âmes de morts.

-- de celle, enfin, en partie sacrificielle, il est vrai, du cheval qui a servi à transporter le cadavre de son maître jusqu'au lieu de sépulture et doit être incinéré avec lui, pour être sa monture dans l'au-delà (sort autrefois partagé par la femme et les serviteurs du mort).

Une mise à mort symbolique retient l'attention : celle du chaman, durant son apprentissage. En effet, 1'apprenti-chaman est soumis symbolịquement à une épreuve consistant en un dépeçage de son corps, suivi d'un examen de ses os, et d'une cuisson de sa chair (par ébullition). Cette épreuve évoque le sort réservé aux victimes sacrificielles, obéissant aussi au schéma de mort et renaissance, qui est explicite lors de 1a "prise d'habit" (27) du chaman : celui-ci y est tenu pour un nouveauné dont doit être coupé le cordon ombilical. Il lui faudra ensuite mettre une grande précaution à récupérer, à la fin de leur cuisson, ses propres morceaux de chair, car il est indispensable, comme dans le sacrifice, de respecter 1 'intégrité constitutive des êtres. Cependant, les rôles s'inversent dans l'apprentissage et la "prise d'habit" du chaman, par rapport au sacrifice : surnaturels y sont les bourreaux, humains les dédicataires, mais les bénêficiaires restent les humains (c'est le seul cas d'identité entre bénéficiaire et dédicataire). Les humains feront un usage réitéré de cette ex-victime qui passera sa vie à s'offrir comme entremetteur

27. Cette expression a été préférée au terme, plus usuel, de consécration, pour éviter toute confusion avec la consécration des animaux, présentée en VII. Ce choix s'accorde mieux, en outre, avec la terminologie bouriate, žodoo barixa, "saisir 1'écorce d'épicéa", emblème de la fonction chamanique (cf. Annuaire de $1^{\prime}$ E.P.H.E., $5^{\circ}$ section, 1976-1977) et ongolxo (cf. page suivante). 
avec ses anciens bourreaux.

Faute de pouvoir faire ici un parallèle plus approfondi, on se bornera à relever que, célestes ou terrestres, les bourreaux font cuire la chair de leurs victimes, car seule une chair cuite peut passer d'un monde à l'autre (28). Toutes les incursions de chair crue hors de leur monde d'origine sont pathogènes : celle du chef des quarante-quatre cieux noirs de l'Est, dont la chute sur terre des membres épars, à la suite d'un combat avec les cieux de l'Ouest, provoque l'apparition de tous les maux de la terre; celles des morts irréguliers qui, faute de pouvoir être incinérés comme tout le monde, sèment le trouble ça et là. Comme la cuisson des morts rituels, l'incinération des morts profanes garantit l'irrêversibilité.

$$
\begin{array}{rl}
V I I . \quad U N & T R A I T E M E N T \quad R I T U E L \\
& D E S \quad \hat{E} T R E S \quad V I V A N T S
\end{array}
$$

Ce qui, de tous les rituels comportant des opérations sur les êtres vivants, fournit les éléments de comparaison les plus fructueux avec le sacrifice est le traitement des animaux appelé consécration. ongolxo, qui désigne ce rite, est un verbe actif formé sur ongon, terme dérivé d'une racine signifiant "barque" et s'appliquant à tout support d'être symbolique, et par contiguité à l'être symbolique lui-même. Ainsi, sont doublement ongon les poupées de feutre ou de bois que 1 'on invoque, oint de beurre et abreuve d'alcool, ou que 1'on gifle et jette lorsque les êtres qui en font leur pied-à-terre se sont mal conduits. Rendre ongon un animal est donc en faire le support d'un esprit (29). Les

28. "A prêsent -- disent les ancêtres --, nous allons découper ta chair et la faire bouillir; tu seras gisant, mort; puis nous te remettrons ta chair en place et tu revivras, tu deviendras chaman. Comme ta chair ne sera pas seule à bouillir, il faudra que tu la reconnaisses, car si nous remettons celle d'un autre cela fera des dêgâts !" (Ksenofontov, 101). Par ailleurs, 1'ébullition est le procédé culinaire qui rappelle le mieux la maturation intra-utérine.

29. Ce que ne voit pas tout à fait Sanžeev (937-938); au cours de sa critique, au demeurant fort pertinente, de la traduction par "consacrer", compris au sens de "vouer". 
espèces qui jouissent de cette faveur sont en premier lieu les bovins, puis les chevaux et exceptionnellement les caprins. L'animal consacré est toujours, et sans ambiguité, le mâle entier (30). Cela lui vaut de n'être ni tondu ni monté (surtout par les femmes), ni battu (au cas où il ferait des siennes dans le troupeau), ni vendu; et, pour un cheval, de n'avoir ni la queue ni la crinière coupées jusqu'à sa mort. Selon les régions, on a coutume soit de le tuer pour le remplacer par un plus jeune, par souci de fécondité, soit de le laisser mourir de sa belle mort, en conservant, fixés sur un poteau devant la yourte ou 1 'enclos son huuraj et ses organes génitaux, celui-ci ayant valeur symbolique de vie, ceux-1à de fécondité.

Le taureau consacré est le représentant du taureau bleu Buca-nojon, "seigneur-taureau", l'ancêtre mythique des Bouriates, à l'origine non seulement de l'ethnie, mais aussi de nombreux rites -- en particulier de ceux de naissance et de fécondité. Le cheval consacré est le plus souvent celui du chaman, mais ce qu'il représente n'est pas clair ; peut-être sont-ce les esprits qui favorisent ses déplacements symboliques. Quant au bouc consacré, il n'en est guère d'exemple que pour le culte rendu à Hüme, l'une de ces âmes frustrées et vengeresses évoquées plus haut, qui sévit en pays Alar'. On rapporte aussi que dans les familles dont les enfants meurent en bas âge, les parents consacrent un bouc qu'ils attachent au berceau de leur dernier-né (Manžigeev, 186).

Les animaux consacrés apparaissent comme une contrepartie des animaux sacrifiés : ceux-ci représentent une prestation périodique réelle, fournie à des troupeaux symboliques dont les maîtres surnaturels, par leur prêsence symbolique permanente dans le troupeau réel sous la forme d'animaux réels (les consacrés), veillent à la protection et à la fécondité des hommes et des bêtes. Le sacrifié meurt réellement pour être, après transfert, symboliquement vivant. Le consacré continue de vivre réellement d'une vie symbolique. Alors que les sacrifiés montent

30. Ce qui ne saurait surprendre, puisqu'il est destiné à assurer symboliquement la reproduction. Ces quelques données et leur analyse rejoignent leurs homologues tuva, présentés par A. Popova (126-127), qui voit les animaux châtrés comme bons pour la communication et les animaux entiers comme bons pour la reproduction. 
des hommes vers les esprits, les consacrés représentent une descente des esprits vers les hommes. Entre hommes et esprits, ou plutôt entre viants et morts claniques, par le jeu du sacrifice et de la consécration, une boucle est bouclée. Mais malgré tous les efforts des hommes pour contraindre les esprits à la bonne conduite, une part de responsabilité reste, non maîtrisable, entre les mains de ces êtres symboliques aussi peu fiables que les humains qu'ils étaient précédemment, ce qui secrète 1 'angoisse permanente.

C'est un circuit comparable à celui du sacrifice et de la consécration qui se réalise dans la personne du chaman. D'abord sacrifié dans la surnature, il est ensuite, en quelque sorte "consacré", sous forme terrestre et permanente, pour le bonheur des siens. Alors que la boucle sacrifice-consécration se présente comme un circuit de tractations sur le capital d'un clan en âmes animales et humaines -- tractations menées entre les vivants et les morts du clan --, la boucle réalisée dans 1a destinẻe chamanique correspond aux dêmarches en tous sens du seul agent de ces tractations.

Quelques remarques, pour finir, sur l'originalité du sacrifice bouriate. A coup sûr, il n'est fait ni pour procurer un aliment aux esprits, ni pour servir de support à la divination. Il $n$ 'est pas non plus, comme le sacrifice hindou, une répétition d'un sacrifice divin initial ; bien au contraire, la première fois qu'un être cêleste fut détruit, ce fut 1'apparition des maux sur terre. De cette catastrophique chute divine, la cuisson du chaman apparaît comme symetrique et inverse : en effet, le chaman est dépecé au ciel au lieu de choir sur terre, et son intervention est orthogène au lieu d'être pathogène. Mais la dëmarche divine et la démarche chamanique sont transgressives et novatrices (ou rénovatrices), alors que les sacrifices sont normatifs et conservateurs (31).

A 1 'encontre du sacrifice hindou aussi, l'exactitude de l'observance liturgique ne suffit pas à assurer l'efficacité; sur le

31. Cette formulation un peu schématique reflète l'état de désagrégation du chamanisme de la fin du XIXe siècle. Le contact avec une société d'ordre et une religion de salut 1 'ont confiné dans les opérations réparatrices, qui s'accomodent de toutes les doctrines. 
déroulement du sacrifice bouriate pèse toujours la possibilitê de l'échec, dû aux moeurs mouvantes d'êtres surnaturels aux défauts humains, et donc pèse l'angoisse. Ceci suggère l'importance de la médiation entre hommes et esprits, et de son agent, le chaman. Le rôle fondamental de ce dernier étant d'écarter les désordres, on comprend que son intervention soit indispensable dans les sacrifices accomplis en réponse à des désordres, et accessoire dans les sacrifices claniques garantissant 1 'ordre.

Les nomades évoqués auront sans doute pondéré le privilège sacrificiel attribué par Mauss aux sociétês agricoles sédentaires, et auront mis en lumière le rapport qui existe entre leur mode de vie et 1 'orientation de l'hospitalité (et du sacrifice) qu'ils valorisent : l'offrande donnée à l'autre chez celui-ci est la plus respectueuse. Par ailleurs, la similitude des modèles d'hospitalité et de sacrifice est à rapporter à la conception de la continuité qui relie les êtres symboliques aux humains.

Transfert d'énergie, certes, le sacrifice bouriate l'est comme les autres. Par le jeu couplé du sacrifice clanique et de la consécration, l'énergie se conserve en circulant à l'intérieur du patrimoine clanique. En revanche, passant, dans la "rançon", d'un support à un autre, sous l'effet d'une double démarche transgressive (32), elle ne fait que mettre en sursis sa déperdition. Son transfert n'assure jamais un profit, tout au plus une balance.

32. Passant de la victime anirnale ou humaine au bénéficiaire, sous 1 'effet de la transgression des catégories symboliques que représente toute "séance" chamanique, et de la transgression de la solidarité sociale et familiale que représente la remise d'une rançon humaine. 


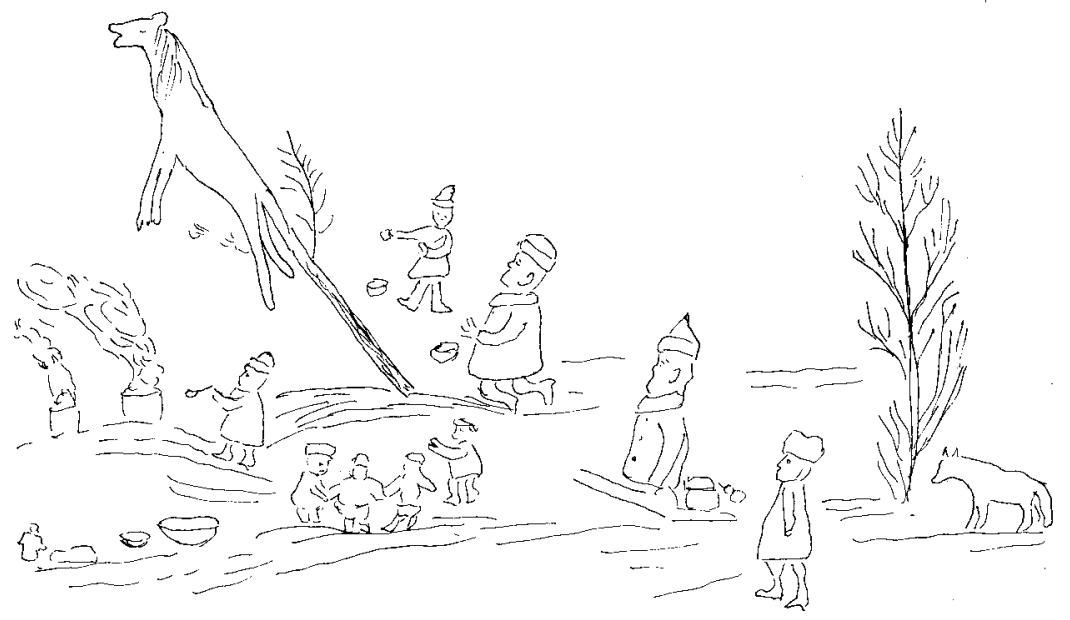


espace sacrificiel

initiative

résidence des esprits

côtê humain : clan

sacrifiant

sacrifié

traitement du sacrifié

dèdicataire

caractéristiques du sacrifice

moyens

objectifs duite de ses anciens;

= bénéficiaire

victime animale 1 'ordre nique résidence des hommes

surnature

côté surnaturel : marginaux (combinaison du droit dynastique réaffirmé par la surnature et du charisme individuel)

communauté, sous la con-

chair bouillie, os incinérés, dépouille offerte

dignes ancêtres collectivement ; représentants de

régulier, communautaire, préventif, propitiatoire, liê à l'institution cla-

démarche normative pour échange positif avec les légitimes de la surnature

maintien de 1 'ordre implicite, fécondité, prospérité ancêtres chamans, dans la surnature

\section{= bénéficiaire}

animal réel ou humain symbolique substitué à humain

chair bouillie, os incinérés, dépouille offerte

indignes anti-ancêtres

individuellement ; reprêsentants de la subversion et fauteurs de désordres

occasionnel, privé, curatif, conjuratoire, expiatoire, 1 ié à 1 'institution chamanique

humain, apprenti-chaman

chair bouillie, os dénombrés port d'un costume spécial lors des "séances" chamaniques

humains = bénéficiaires ; = victimes des désordres

obligatoire une fois pour tout chaman ; privé, en prélude à "prise d'habit" devant la communauté

démarche transgressive pour neutralisation des marginaux de la surnature

éloignement des désordres rétablissement du statu quo instauration d'une entremise symbolique permanente, orthogène 
sacrifice

prestation périodique réelle

d'animal réel

à troupeau symbolique

appartenant à maîtres surnaturels

témoignant de prospérité

1 'animal meurt réellement

pour être transféré comme vivant

symbolique

monte des homes vers les esprits consécration

présence permanente symbolique dans animal réel

de troupeau réel terrestre

de maîtres surnaturels

assurant reproduction et production

1 'animal vit réellement

d'une vie symbolique d'esprit se substituant à sa vie d'animal

descend des esprits vers les hommes

Tableau 2.

$R \mathscr{E} F E R E N C E S$

BALDAEV, S. P., 1970. RodosZovnye predanija i Zegendy Burjat.č. 1. Bulagaty i Exirity. Ulan-Ude, Burjatskij institut obščestvennyx nauk AN SSSR, $363 \mathrm{p}$.

---, 1975. "Objets descendus" et "pierres écrites" dans le culte populaire bouriate. Etudes mongoles, 6, pp. 161-181.

BURDUKOV, A. V., 1927. Čelovečeskie žertvoprinošenija u sovremmennyx Mongolov. Sibirskie ogni, III, pp. 184-189.

ČISTOXIN, I., 1896. Dve Šamanskie molitvy proiznosimye na tajlaganax u Burjat. Izvestija Vostoćno-sibirskogo otdela Russkogo Geograficeskogo Obšéestva (VSORGO), XXVI, 4-5, pp. 282-283.

GIRČENKO, V. P., 1927. Kudinskie tajlgany. Burjatievedenie, 3-4, pp. 7378.

HAMAYON, R., 1975. L'os distinctif et la chair indifférente. Études mongoles, 6, pp. 99-122.

---, 1977. I1 n'y a pas de fumée sans dieu. L'Ethnographie, n. s., 7475, pp. 105-122.

Histoire secrète des Mongols, 1949. Restitution et traduction par Paul

Pelliot. Paris, A. Maisonneuve, $197 \mathrm{p}$.

KSENOFONTOV, G. F., 1929. Xrestes. Śamanizm $i$ xristianstvo. Irkutsk, ix-143 p.

LIGETI, L., 1973. Le sacrifice offert aux ancêtres dans 1'Histoire secrète des Mongols. Acta Orientalia Hungaricae, XXVII, 2, pp. 145-161.

MANŽIGEEV, I. A., 1960. Jangutskij burjatskij rod. Ulan-Ude, Burjatskij 
kompleksnj naučno-issledovatel'skij institut AN SSSR, 232 p.

MIXAJLOV, T. M., 1962. Samanstvo $i$ ego perezitki. Irkutsk, 37 p. irkutskoe oblastnoe otdelenie obšcestva po rasprostraneniju političeskix i naucnyx znanij.

MIXAJLOV, T. M. et XORoŠIX, P. P., 1973. Burjatskij šamanizm. Ukazatel' literatury (1774-1971 gg.), Ulan-Ude, Burjatskij institut obščestvennyx nauk AN SSSR.

ORDYNSKIJ, A. K., 1896. Ođerki burjatskoj žizni. (Zapiski moego znakomogo). Tobol'sk, 119 p.

POPOVA, A., 1977. La chevauchée nocturne du cavalier invisible, L'Ethnographie, $\mathrm{n}^{\circ}$ 74-75, pp. 101-128.

ROCKHILL, W. W., 1900. The Joumey of Wizliam of Rubmuck to the Eastem Parts of the World, 1253-1255, as narrated by himself, with two accounts of the earlier journey of John of Pian de Carpine. Trans. from the latin and ed. by w.w. Rockhi11. The Hakluyt Society, second series, $n^{\circ}$ IV. Kraus reprint limited, Nendeln/Liechtenstein, 1967, 1vi-304 p., carte dépl.

SANŽEEV, G. D., 1927-1928. Weltanschauung und Schamanismus der AlarenBurjaten. Anthropos, 22, pp. 576-613; 933-955 ; 23, pp. 538-560 ; 967-986.

VJATKINA, K. V., 1969. Očerki kuZ'tury $i$ byta Burjat. Leningrad, Nauka $217 \mathrm{p}$.

XANGALOV, M. N., 1958-1960. Sobranie sočinenij, 3 vo1. (recueil d'articles publiés entre 1888 et 1910). Ulan-Ude, Burjatskij kompleksnyj naučno-isledovatel'skij institut AN SSSR.

ŽUKOVSKAJA, N. L., 1977. Lamaism i rannye formy religii. Moscou, Nauka, $199 \mathrm{p}$. 